\title{
Sepsis Presenting in Hospitals versus Emergency Departments: Demographic, Resuscitation, and Outcome Patterns in a Multicenter Retrospective Cohort
}

\author{
Daniel E Leisman, BS ${ }^{1,2,3 *}$; Catalina Angel, MPA'; Sandra M Schneider, MD²,4 \\ Jason A D'Amore, MD²; John K D’Angelo, MD²; Martin E Doerfler, MD ${ }^{5,6}$
}

${ }^{1} /$ cahn School of Medicine at Mount Sinai, New York, New York; ${ }^{2}$ Department of Emergency Medicine, Hofstra-Northwell School of Medicine, Hempstead, New York; ${ }^{3}$ Global Sepsis Alliance, Jena, Germany; ${ }^{4}$ American College of Emergency Physicians, Irving, Texas; ${ }^{5}$ Department of Medicine, Hofstra-Northwell School of Medicine, Hempstead, New York; ${ }^{6}$ Department of Science Education, Hofstra-Northwell School of Medicine, Hempstead, New York.

BACKGROUND: Differences between hospital-presenting sepsis (HPS) and emergency department-presenting sepsis (EDPS) are not well described.

OBJECTIVES: We aimed to (1) quantify the prevalence of HPS versus EDPS cases and outcomes; (2) compare HPS versus EDPS characteristics at presentation; (3) compare HPS versus EDPS in process and patient outcomes; and (4) estimate risk differences in patient outcomes attributable to initial resuscitation disparities.

DESIGN: Retrospective consecutive-sample cohort.

SETTING: Nine hospitals from October 1, 2014, to March 31, 2016.

PATIENTS: All hospitalized patients with sepsis or septic shock, as defined by simultaneous (1) infection, (2) $\geq 2$ Systemic Inflammatory Response Syndrome (SIRS) criteria, and (3) $\geq 1$ acute organ dysfunction criterion. EDPS met inclusion criteria while physically in the emergency department (ED). HPS met the criteria after leaving the ED.

MEASUREMENTS: We assessed overall HPS versus EDPS contributions to case prevalence and outcomes, and then compared group differences. Process outcomes included 3-hour bundle compliance and discrete bundle elements (eg, time to antibiotics). The primary patient outcome was hospital mortality.
RESULTS: Of 11,182 sepsis hospitalizations, 2,509 (22.4\%) were hospital-presenting. HPS contributed 785 (35\%) sepsis mortalities. HPS had more frequent heart failure (OR: 1.31, Cl: 1.18-1.47), renal failure (OR: 1.62, Cl: 1.38-1.91), gastrointestinal source of infection (OR: 1.84, Cl: 1.48-2.29), euthermia (OR: 1.45, Cl: 1.10-1.92), hypotension (OR: 1.85, $\mathrm{Cl}: 1.65-2.08)$, or impaired gas exchange (OR: $2.46, \mathrm{Cl}$ : 1.43-4.24). HPS were admitted less often from skilled nursing facilities (OR: 0.44, Cl: 0.32-0.60), had chronic obstructive pulmonary disease (OR: $0.53, \mathrm{Cl}: 0.36-0.78)$, tachypnea (OR: $0.76, \mathrm{Cl}: 0.58-0.98)$, or acute kidney injury (OR: 0.82 , Cl: $0.68-0.97)$. In a propensity-matched cohort $(n=3,844)$, HPS patients had less than half the odds of 3-hour bundle compliant care (17.0\% vs $30.3 \%$, OR: $0.47, \mathrm{Cl}: 0.40-0.57)$ or antibiotics within three hours (66.2\% vs $83.8 \%$, OR: 0.38 , Cl: $0.32-0.44)$ vs EDPS. HPS was associated with higher mortality (31.2\% vs $19.3 \%$, OR: $1.90, \mathrm{Cl}: 1.64-2.20) ; 23.3 \%$ of this association was attributable to differences in initial resuscitation (resuscitation-adjusted OR: 1.69, Cl: 1.43-2.00).

CONCLUSIONS: HPS differed from EDPS by admission source, comorbidities, and clinical presentation. These patients received markedly less timely initial resuscitation; this disparity explained a moderate proportion of mortality differences. Journal of Hospital Medicine 2019;14:340-348. Published online first April 8, 2019. () 2019 Society of Hospital Medicine epsis is both the most expensive condition treated and the most common cause of death in hospitals in the United States. ${ }^{1-3}$ Most sepsis patients (as many as $80 \%$ to $90 \%$ ) meet sepsis criteria on hospital arrival, but mortality and costs are higher when meeting criteria after admission. ${ }^{3-6}$ Mechanisms of this increased mortality for these distinct populations are not well explored. Patients who present septic in the emergency department (ED) and patients who

*Corresponding Author: Daniel E Leisman, BS; E-mail: deleisman@gmail.com; Telephone: 516-941-8468.

Find Additional Supporting Information in the online version of this article.

Received: October 16, 2018; Revised: January 17, 2019;

Accepted: February 2, 2019

๑) 2019 Society of Hospital Medicine DOI 10.12788/jhm.3188 present as inpatients likely present very different challenges for recognition, treatment, and monitoring.? Yet, how these groups differ by demographic and clinical characteristics, the etiology and severity of infection, and patterns of resuscitation care are not well described. Literature on sepsis epidemiology on hospital wards is particularly limited. ${ }^{8}$

This knowledge gap is important. If hospital-presenting sepsis (HPS) contributes disproportionately to disease burd$\mathrm{CHFens}$, it reflects a high-yield population deserving the focus of quality improvement (Ql) initiatives. If specific causes of disparities were identified - eg, poor initial resuscitation - they could be specifically targeted for correction. Given that current treatment guidelines are uniform for the two populations, ${ }^{9,10}$ characterizing phenotypic differences could also have implications for both diagnostic and therapeutic recommendations, particularly if the groups display substantially differing clinical 
presentations. Our prior work has not probed these effects specifically, but suggested ED versus inpatient setting at the time of initial sepsis presentation might be an effect modifier for the association between several elements of fluid resuscitation and patient outcomes. ${ }^{11,12}$

We, therefore, conducted a retrospective analysis to ask four sequential questions: (1) Do patients with HPS, compared with EDPS, contribute adverse outcome out of proportion to case prevalence? (2) At the time of initial presentation, how do HPS patients differ from EDPS patients with respect to demographics, comorbidities, infectious etiologies, clinical presentations, and severity of illness (3) If holding observed baseline factors constant, does the physical location of sepsis presentation inherently increase the risk for treatment delays and mortality? (4) To what extent can differences in the likelihood for timely initial treatment between the ED and inpatient settings explain differences in mortality and patient outcomes?

We hypothesized a priori that HPS would reflect chronically sicker patients whom both received less timely resuscitation and who contributed disproportionately frequent bad outcomes. We expected disparities in timely resuscitation care would explain a large proportion of this difference.

\section{METHODS}

We performed a retrospective analysis of the Northwell Sepsis Database, a prospectively captured, multisite, real world, consecutive-sample cohort of all "severe sepsis" and septic shock patients treated at nine tertiary and community hospitals in New York from October 1, 2014, to March 31, 2016. We analyzed all patients from a previously published cohort. ${ }^{11}$

\section{Database Design and Structure}

The Northwell Sepsis Database has previously been described in detail. 11,13,14 Briefly, all patients met clinical sepsis criteria: (1) infection AND (2) $\geq 2$ (SIRS) criteria AND (3) $\geq 1$ acute organ dysfunction criterion. Organ dysfunction criteria were hypotension, acute kidney injury (AKI), coagulopathy, altered gas exchange, elevated bilirubin $(\geq 2.0 \mathrm{mg} / \mathrm{dL})$, or altered mental status (AMS; clarified in Supplemental Table 1). All organ dysfunction was not otherwise explained by patients' medical histories; eg, patients on warfarin anticoagulation were not documented to have coagulopathy based on international normalized ratio $>1.5$. The time of the sepsis episode (and database inclusion) was the time of the first vital sign measurement or laboratory result where a patient simultaneously met all three inclusion criteria: infection, SIRS, and organ dysfunction. The database excludes patients who were $<18$ years, declined bundle interventions, had advance directives precluding interventions, or were admitted directly to palliative care or hospice. Abstractors assumed comorbidities were absent if not documented within the medical record and that physiologic abnormalities were absent if not measured by the treatment team. There were no missing data for the variables analyzed. We report analysis in adherence with the STROBE statement guidelines for observational research.

\section{Exposure}

The primary exposure was whether patients had EDPS versus HPS. We defined EDPS patients as meeting all objective clinical inclusion criteria while physically in the ED. We defined HPS as first meeting sepsis inclusion criteria outside the ED, regardless of the reason for admission, and regardless of whether patients were admitted through the ED or directly to the hospital. All ED patients were admitted to the hospital.

\section{Outcomes}

Process outcomes were full 3-hour bundle compliance, time to antibiotic administration, blood cultures before antibiotics, time to fluid initiation, the volume of administered fluid resuscitation, lactate result time, and whether repeat lactate was obtained (Supplemental Table 2). Treatment times were times of administration (rather than order time). The primary patient outcome was hospital mortality. Secondary patient outcomes were mechanical ventilation, ICU admission, ICU days, hospital length of stay (LOS). We discounted HPS patients' LOS to include only days after meeting the inclusion criteria. Patients were excluded from the analysis of the ICU admission outcome if they were already in the ICU prior to meeting sepsis criteria.

\section{Statistical Analysis}

We report continuous variables as means (standard deviation) or medians (interquartile range), and categorical variables as frequencies (proportions), as appropriate. Summative statistics with 95\% confidence intervals $(\mathrm{Cl})$ describe overall group contributions. We used generalized linear models to determine patient factors associated with EDPS versus HPS, entering random effects for individual study sites to control for intercenter variability.

Next, to generate a propensity-matched cohort, we computed propensity scores adjusted from a priori selected variables: age, sex, tertiary versus community hospital, congestive heart failure (CHF), renal failure, COPD, diabetes, liver failure, immunocompromise, primary source of infection, nosocomial source, temperature, initial lactate, presenting hypotension, altered gas exchange, AMS, AKI, and coagulopathy. We then matched subjects 1:1 without optimization or replacement, imposing a caliper width of 0.01 ; ie, we required matched pairs to have a $<1.0 \%$ difference in propensity scores. The macro used to match subjects is publically available. ${ }^{15}$

We then compared resuscitation and patient outcomes in the matched cohort using generalized linear models, ie, doubly-robust estimation (DRE). ${ }^{16}$ When assessing patient outcomes corrected for resuscitation, we used mixed DRE/multivariable regression. We did this for two reasons: first, DRE has the advantage of only requiring only one approach (propensity vs covariate adjustments) to be correctly specified. ${ }^{16}$ Second, computing propensity scores adjusted for resuscitation would be inappropriate given that resuscitation occurs after the exposure allocation (HPS vs EDPS). However, these factors could still impact the outcome and in fact, we hypothesized they were potential mediators of the exposure effect. To interrogate this mediating relationship, we recapitulated the DRE model- 
TABLE 1. Selected Patient Characteristics and Outcomes in Unmatched and Matched Cohorts

\begin{tabular}{|c|c|c|c|c|c|}
\hline Variable & \multicolumn{3}{|c|}{ Entire (Unmatched) Cohort } & \multicolumn{2}{|c|}{ Matched Cohort } \\
\hline $\mathrm{N}$ & 11,182 & $8,673(77.6 \%)$ & $2,509(22.4 \%)$ & $1,942(50.0 \%)$ & $1,942(50.0 \%)$ \\
\hline \multicolumn{6}{|l|}{ Demographics } \\
\hline Age $^{*}-$ median (IQR) & $74(62,85)$ & $75(62,85)$ & $73(62,83)$ & $73(61-84)$ & $74(62-84)$ \\
\hline Male Sex* & $5,740(51.3 \%)$ & $4,436(51.1 \%)$ & $1,304(52.0 \%)$ & $1,025(52.8 \%)$ & $1,021(52.6 \%)$ \\
\hline \multicolumn{6}{|l|}{ Comorbidities $^{\mathrm{a}}$} \\
\hline Heart Failure & $1,647(14.7 \%)$ & $1,171(13.5 \%)$ & $476(19.0 \%)$ & $322(16.6 \%)$ & $351(18.1 \%)$ \\
\hline Renal Failure & $1,161(10.4 \%)$ & $754(8.7 \%)$ & $407(16.2 \%)$ & $278(14.3 \%)$ & $284(14.6 \%)$ \\
\hline $\mathrm{COPD}^{*}$ & $793(7.1 \%)$ & $666(7.7 \%)$ & $127(5.1 \%)$ & $96(4.9 \%)$ & $100(5.1 \%)$ \\
\hline Immune modifying medications & $2,346(21.0 \%)$ & $1,748(20.2 \%)$ & $598(23.8 \%)$ & $439(22.6 \%)$ & $459(23.6 \%)$ \\
\hline Skin/Soft Tissue Infection Source & $778(7.0 \%)$ & $644(7.4 \%)$ & $134(5.3 \%)$ & $154(7.9 \%)$ & $92(4.7 \%)$ \\
\hline Gastrointestinal Infection Source & $1,071(9.6 \%)$ & $734(8.5 \%)$ & $337(13.4 \%)$ & $202(10.4 \%)$ & $268(13.8 \%)$ \\
\hline Other/Unknown Infection Source & $2,071(18.5 \%)$ & $1,518(17.5 \%)$ & $553(22.0 \%)$ & $454(23.4 \%)$ & $426(21.9 \%)$ \\
\hline Confirmed Nosocomial Source & $1,213(10.9 \%)$ & $705(8.1 \%)$ & $508(20.3 \%)$ & $339(17.5 \%)$ & $332(17.1 \%)$ \\
\hline Fever* & $4,040(36.1 \%)$ & $3,334(38.4 \%)$ & $706(28.1 \%)$ & $637(32.8 \%)$ & $553(28.5 \%)$ \\
\hline Leukocytosis & $6,596(59.0 \%)$ & $5,146(59.3 \%)$ & $1,450(57.8 \%)$ & $1,123(57.8 \%)$ & $1,128(58.1 \%)$ \\
\hline \multicolumn{6}{|l|}{ Severity of IIIness } \\
\hline Initial Lactate (mmol/L)-mean (SD) & $3.2(2.4)$ & $3.3(2.3)$ & $3.1(2.7)$ & $3.1(2.2)$ & $3.1(2.7)$ \\
\hline Hypotension & $3,714(33.2 \%)$ & $2,551(29.4 \%)$ & 1,163 (46.4\%) & $872(44.9 \%)$ & $849(43.7 \%)$ \\
\hline Altered Gas Exchange $e^{b}$ & $2,412(21.6 \%)$ & $1,606(18.5 \%)$ & $806(32.1 \%)$ & $604(31.1 \%)$ & $622(32.0 \%)$ \\
\hline Antibiotics within $3 \mathrm{~h}$ & $9,040(80.8 \%)$ & $7,437(85.7 \%)$ & $1,603(63.9 \%)$ & $1,628(83.8 \%)$ & $1,285(66.2 \%)$ \\
\hline Antibiotics within $6 \mathrm{~h}$ & $9,987(89.3 \%)$ & $8,111(93.5 \%)$ & $1,876(74.8 \%)$ & $1,796(92.5 \%)$ & $1,496(77.0 \%)$ \\
\hline Blood Cultures Before Antibiotics & $7,350(67.3 \%)$ & $6,170(71.1 \%)$ & $1,360(54.2 \%)$ & $1,350(69.5 \%)$ & $1,036(53.3 \%)$ \\
\hline Time to Fluid Initiation-mean (SD) & $118(149)$ & $86(128)$ & $220(160)$ & $89(129)$ & $210(166)$ \\
\hline Fluid Volume (mL/kg)-mean (SD) & $22.9(18.7)$ & $25.4(18.4)$ & $14.1(16.9)$ & $26.0(18.8)$ & $15.4(17.2)$ \\
\hline \multicolumn{6}{|l|}{ Patient Outcomes } \\
\hline In-Hospital Mortality & $2,241(20.0 \%)$ & $1,456(16.8 \%)$ & $785(31.3 \%)$ & $374(19.3 \%)$ & $605(31.2 \%)$ \\
\hline Mechanical Ventilation & $3,265(29.2 \%)$ & $2,024(23.3 \%)$ & $1,241(49.5 \%)$ & $532(27.4 \%)$ & $1,000(51.5 \%)$ \\
\hline
\end{tabular}

All data presented as frequency (percentage) unless otherwise indicated. Full tabulation of patient characteristics and outcomes is available in the online supplement.

*Indicates variable was used in generating propensity score for matching.

${ }^{a}$ Comorbidities reflect status at time zero and would not reflect conditions developing subsequently during hospital stay.

${ }^{b}$ Altered Gas Exchange defined as $\mathrm{PaO} 2 / \mathrm{FiO} 2<300$ or an increased $\mathrm{O} 2$ requirement to maintain $\mathrm{SaO} 2>90 \%$.

'Acute Kidney Injury defined as creatinine $>2.0$ or $50 \%$ increase from a known baseline.

Abbreviations: COPD, chronic obstructive pulmonary disease; EDPS, emergency department-presenting sepsis; HPS, hospital-presenting sepsis, ICU, intensive care unit; IQR, interquartile range; SNF, skilled nursing facility; SD, standard deviation.

ing but added covariates for resuscitation factors. Resuscitation-adjusted models controlled for timeliness of antibiotics, fluids, and lactate results; blood cultures before antibiotics; repeat lactate obtained, and fluid volume in the first six hours.
Since ICU days and LOS are subject to competing risks bias (LOS could be shorter if patients died earlier), we used proportional hazards models where "the event" was defined as a live discharge to censor for mortality and we report output as 
TABLE 2. Adjusted Associations of Patient Characteristics with Hospital vs ED-Presenting Sepsis

\begin{tabular}{|c|c|c|c|c|c|c|}
\hline Variable & \multicolumn{3}{|c|}{ (All) Hospital-Presenting Sepsis } & \multicolumn{3}{|c|}{ (Non-ICU) Hospital-Presenting Sepsis } \\
\hline Male Sex & 0.98 & 0.93 to 1.02 & .30 & 1.01 & 0.84 to 1.20 & .96 \\
\hline Age (per 10 years) & 1.04 & 0.86 to 1.27 & .67 & 1.01 & 0.97 to 1.06 & .56 \\
\hline Body Mass Index* & 1.01 & 1.01 to 1.02 & $<.001$ & 1.01 & 1.00 to 1.01 & .069 \\
\hline \multicolumn{7}{|l|}{ Comorbidities } \\
\hline Congestive Heart Failure* $^{*}$ & 1.31 & 1.18 to 1.47 & $<.001$ & 1.27 & 1.12 to 1.44 & $<.001$ \\
\hline Chronic Renal Failure* & 1.62 & 1.38 to 1.91 & $<.001$ & 1.59 & 1.29 to 1.95 & $<.001$ \\
\hline $\mathrm{COPD}^{*}$ & 0.53 & 0.36 to 0.78 & .001 & 0.52 & 0.34 to 0.79 & .003 \\
\hline Diabetes & 0.94 & 0.86 to 1.03 & .20 & 0.90 & 0.80 to 1.01 & .069 \\
\hline Urinary & 0.94 & 0.81 to 1.08 & .37 & 1.03 & 0.87 to 1.20 & .75 \\
\hline Gastrointestinal* & 1.84 & 1.48 to 2.29 & $<.001$ & 1.96 & 1.47 to 2.61 & $<.001$ \\
\hline Skin and Soft Tissue* & 0.73 & 0.55 to 0.97 & .030 & 0.97 & 0.67 to 1.40 & .88 \\
\hline Other/Unknown & 1.37 & 0.87 to 2.15 & .17 & 1.39 & 0.90 to 2.14 & .14 \\
\hline Confirmed Nosocomial Etiology* & 2.61 & 1.19 to 5.71 & .016 & 2.98 & 1.23 to 7.19 & .015 \\
\hline Immunocompromized at Presentation & 1.05 & 0.76 to 1.46 & .77 & 1.08 & 0.83 to 1.41 & .58 \\
\hline Tachycardia $>90$ beats/minute & 0.81 & 0.62 to 1.05 & .11 & 0.88 & 0.70 to 1.11 & .29 \\
\hline Tachypnea $>20$ breaths/minute* & 0.76 & 0.58 to 0.98 & .038 & 0.69 & 0.51 to 0.94 & .017 \\
\hline \multicolumn{7}{|l|}{ Body Temperature (vs Febrile) } \\
\hline Euthermic $\left(>36.0^{\circ} \mathrm{C},<38.3^{\circ} \mathrm{C}\right)^{*}$ & 1.45 & 1.10 to 1.92 & .009 & 1.35 & 1.03 to 1.76 & .030 \\
\hline Altered Gas Exchange* & 2.46 & 1.43 to 4.24 & .001 & 2.35 & 1.42 to 3.90 & .001 \\
\hline Acute Kidney Injury* & 0.82 & 0.68 to 0.97 & .022 & 0.77 & 0.64 to 0.93 & .008 \\
\hline Coagulopathy & 0.92 & 0.53 to 1.57 & .75 & 0.81 & 0.44 to 1.50 & .51 \\
\hline Thrombocytopenia & 1.26 & 0.76 to 2.10 & .38 & 1.28 & 0.74 to 2.19 & .38 \\
\hline
\end{tabular}

Results from two generalized linear models to determine whether sepsis developed after admission or while in the community. Models included the above terms as well as random effects to control for intercenter variability.

Abbreviations: $\mathrm{Cl}$, confidence interval; COPD, chronic obstructive pulmonary disease, SNF, skilled nursing facility.

inverse hazard ratios. We also tested interaction coefficients for discrete bundle elements and HPS to determine if specific bundle elements were effect modifiers for the association between the presenting location and mortality risk. Finally, we estimated attributable risk differences by comparing adjusted odds ratios of adverse outcome with and without adjustment for resuscitation variables, as described by Sahai et al..$^{17}$

As sensitivity analyses, we recomputed propensity scores and generated a new matched cohort that excluded HPS patients who met criteria for sepsis while already in the ICU for another reason (ie, excluding ICU-presenting sepsis). We then recapitu- lated all analyses as above for this cohort. We performed analyses using SAS version 9.4 (SAS Institute, Cary, North Carolina).

\section{RESULTS}

\section{Prevalence and Outcome Contributions}

Of the 11,182 sepsis patients in the database, we classified $2,509(22 \%)$ as HPS (Figure 1). HPS contributed 785 (35\%) of 2,241 sepsis-related mortalities, 1,241 (38\%) mechanical ventilations, and 1,762 (34\%) ICU admissions. Of 39,263 total ICU days and 127,178 hospital days, HPS contributed 18,104 (46.1\%) and 44,412 (34.9\%) days, respectively. 


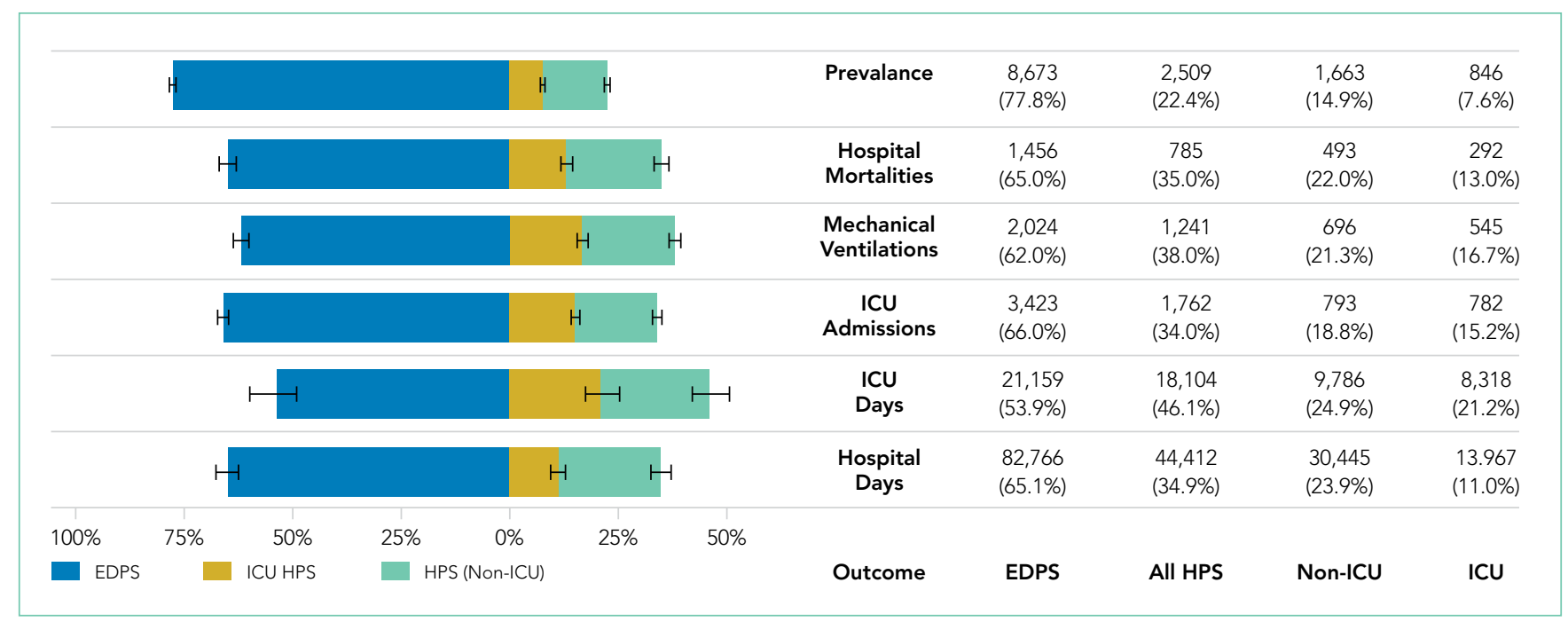

FIG 1. Displays contributions of Hospital vs ED-presenting sepsis to overall case prevalence and patient outcomes. Hospital presentations are also stratified into sepsis presentations that occurred during an ICU admission versus those that occurred outside the ICU on the hospital wards. Brackets indicate $95 \%$ Cls.

Abbreviations: EDPS, ED presenting sepsis; HPS, hospital presenting sepsis; ICU, intensive care unit.

\section{Patient Characteristics}

Most HPS presented early in the hospital course, with 1,352 (53.9\%) cases meeting study criteria within three days of admission. Median time from admission to meeting study criteria for HPS was two days (interquartile range: one to seven days). We report selected baseline patient characteristics in Table 1 and adjusted associations of baseline variables with HPS versus EDPS in Table 2. The full cohort characterization is available in Supplemental Table 3. Notably, HPS patients more often had CHF (aOR [adjusted odds ratio\}: 1.31, Cl: 1.18-1.47) or renal failure (aOR: 1.62, Cl: 1.38-1.91), gastrointestinal source of infection (aOR: 1.84, Cl: 1.48-2.29), hypothermia (aOR: 1.56, Cl: 1.28-1.90) hypotension (aOR: 1.85, Cl: 1.65-2.08), or altered gas exchange (aOR: 2.46, Cl: 1.43-4.24). In contrast, HPS patients less frequently were admitted from skilled nursing facilities (aOR: 0.44, Cl: 0.320.60), or had COPD (aOR: 0.53, Cl: 0.36-0.76), fever (aOR: 0.70, Cl: 0.52-0.91), tachypnea (aOR: 0.76, Cl: 0.58-0.98), or AKI (aOR: 082, $\mathrm{Cl}$ : 0.68-0.97). Other baseline variables were similar, including respiratory source, tachycardia, white cell abnormalities, AMS, and coagulopathies. These associations were preserved in the sensitivity analysis excluding ICU-presenting sepsis.

\section{Propensity Matching}

Propensity score matching yielded 1,942 matched pairs ( $\mathrm{n}=$ $3,884,77 \%$ of HPS patients, $22 \%$ of EDPS patients). Table 1 and Supplemental Table 3 show patient characteristics after propensity matching. Supplemental Table 4 shows the propensity model. The frequency densities are shown for the cohort as a function of propensity score in Supplemental Figure 1. After matching, frequencies between groups differed by $<5 \%$ for all categorical variables assessed. In the sensitivity analysis, propensity matching (model in Supplemental Table 5) resulted in 1,233 matched pairs ( $n=2,466,49 \%$ of HPS patients, $14 \%$ of EDPS patients), with group differences comparable to the primary analysis.

\section{Process Outcomes}

We present propensity-matched differences in initial resuscitation in Figure 2A for all HPS patients, as well as non-ICU-presenting HPS, versus EDPS. HPS patients were roughly half as likely to receive fully 3 -hour bundle compliant care $(17.0 \%$ vs 30.3\%, aOR: 0.47, Cl: 0.40-0.57), to have blood cultures drawn within three hours prior to antibiotics (44.9\% vs $67.2 \%$, aOR: $0.40, \mathrm{Cl}: 0.35-0.46)$, or to receive fluid resuscitation initiated within two hours (11.1\% vs $26.1 \%$, aOR: $0.35, \mathrm{Cl}: 0.29-0.42)$. Antibiotic receipt within one hour was comparable $(45.3 \%$ vs 48.1\%, aOR: 0.89, Cl: 0.79-1.01). However, differences emerged for antibiotics within three hours $(66.2 \%$ vs $83.8 \%$, aOR: 0.38 , $\mathrm{Cl}$ : $0.32-0.44)$ and persisted at six hours $(77.0 \%$ vs $92.5 \%$, aOR: $0.27, \mathrm{Cl}$ : 0.22-33). Excluding ICU-presenting sepsis from propensity matching exaggerated disparities in antibiotic receipt at one hour (43.4\% vs $49.1 \%$, aOR: $0.80, \mathrm{Cl}$ : $0.68-0.93$ ), three hours (64.2\% vs $86.1 \%$, aOR: $0.29, \mathrm{Cl}: 0.24-0.35)$, and six hours (75.7\% vs 93.0\%, aOR: 0.23, Cl: 0.18-0.30). HPS patients more frequently had repeat lactate obtained within 24 hours $(62.4 \%$ vs $54.3 \%$, aOR: $1.40, \mathrm{Cl}: 1.23-1.59)$.

\section{Patient Outcomes}

HPS patients had higher mortality (31.2\% vs19.3\%), mechanical ventilation (51.5\% vs27.4\%), and ICU admission $(60.6 \%$ vs 46.5\%) (Table 1 and Supplemental Table 6). Figure 2b shows propensity-matched and covariate-adjusted differences in patient outcomes before and after adjusting for initial resuscitation. aORs corresponded to approximate relative risk differences ${ }^{18}$ of 1.38 (Cl: 1.28-1.48), 1.68 (Cl: 1.57-1.79), and 1.72 (Cl: 1.61-1.84) for mortality, mechanical ventilation, and ICU admission, respectively. HPS was associated with $83 \%$ longer mortality-censored ICU stays (five vs nine days, $\mathrm{HR}^{-1}: 1.83, \mathrm{Cl}$ : 1.65-2.03), and 108\% longer hospital stay (eight vs 17 days, $\mathrm{HR}^{-1}$ : $2.08, \mathrm{Cl}$ : 1.93-2.24). After adjustment for resuscitation, all effect sizes decreased but persisted. The initial crystalloid 


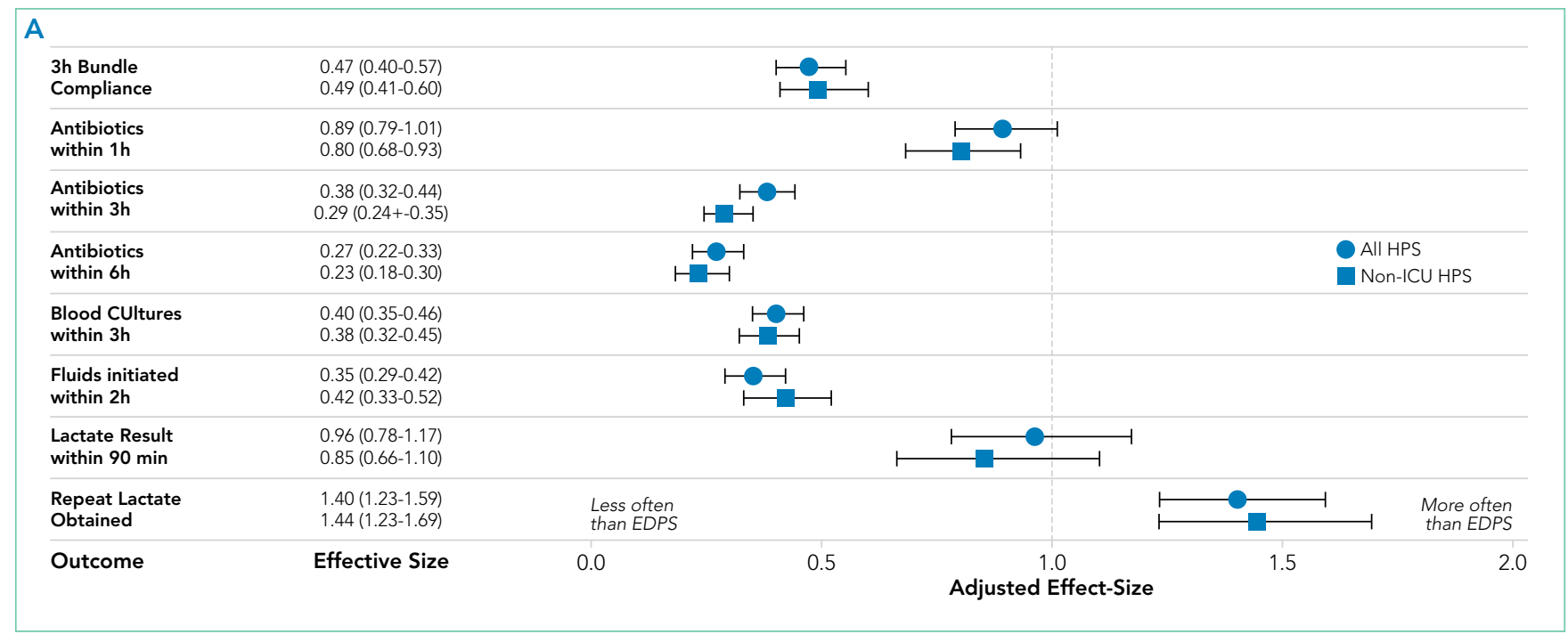

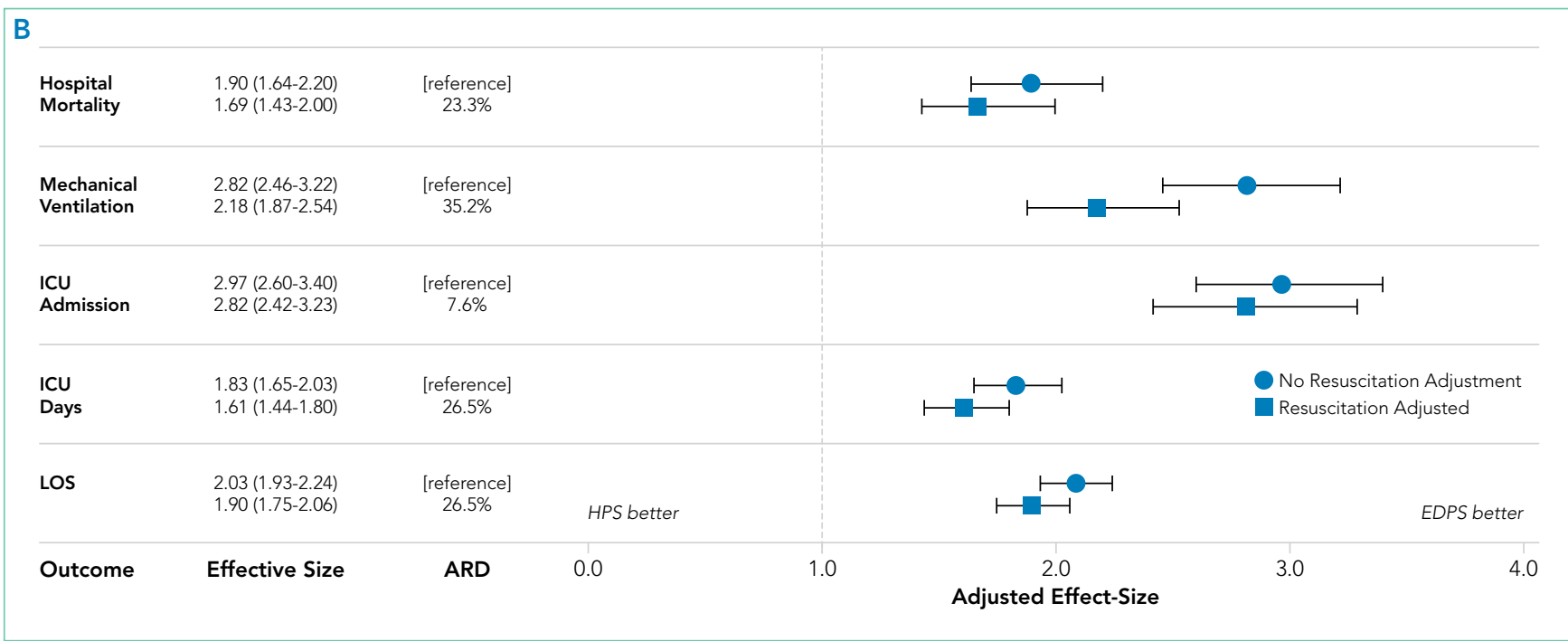

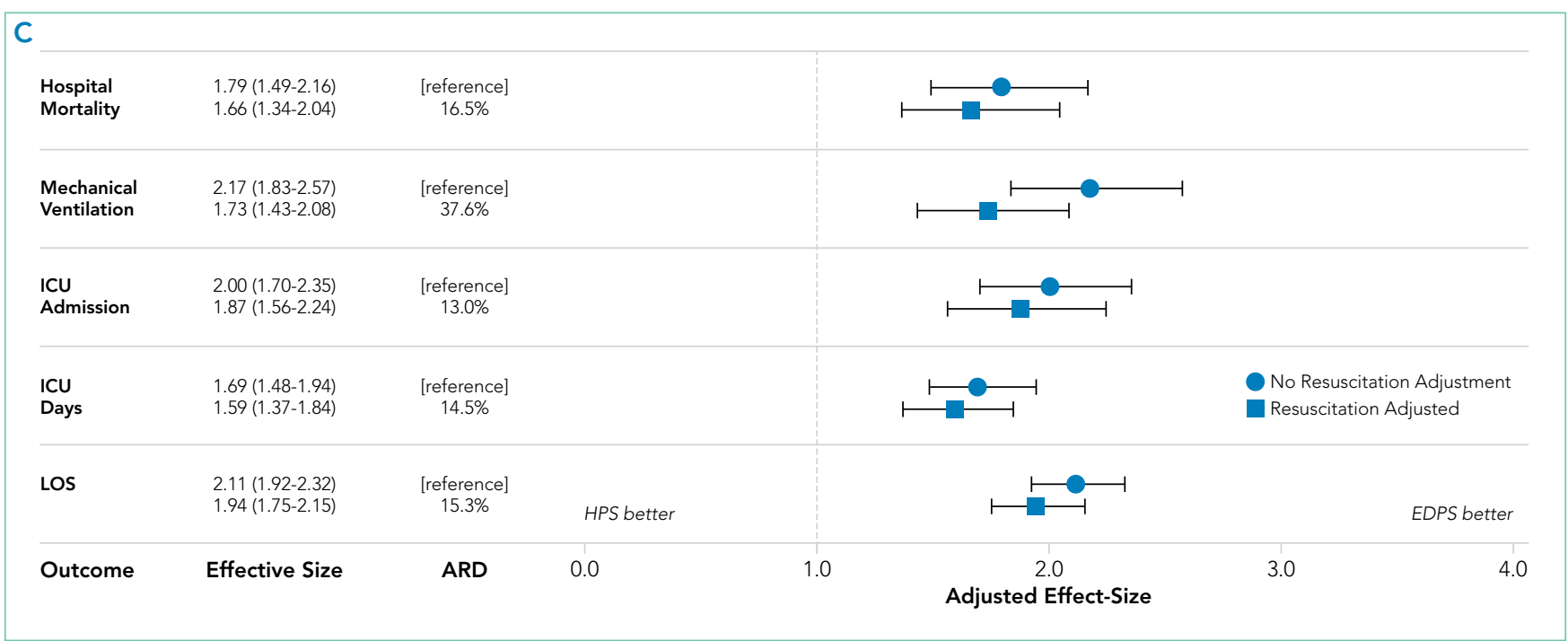

FIG 2. (A) Displays the adjusted likelihood of HPS vs EDPS patients to receive specific initial resuscitation interventions. (B) Displays the adjusted likelihood of all HPS patients to experience the specified outcome, with and without correction for differences in resuscitation delivery. The ARD is the percentage of the outcome difference between HPS and EDPS that was explainable by differences in initial resuscitation delivery. (C) Displays the likelihood of outcome as in (b) but excluding HPS patients whose sepsis presentation occurred in the ICU after admission for another reason. Brackets indicate $95 \% \mathrm{Cls}$.

Abbreviations: ARD, adjusted risk-difference; EDPS, ED presenting sepsis; HPS, hospital presenting sepsis; ICU, intensive care unit. 
volume was a significant negative effect modifier for mortality (Supplemental Table 7). That is, the magnitude of the association between HPS and greater mortality decreased by a factor of 0.89 per $10 \mathrm{~mL} / \mathrm{kg}$ given (Cl: 0.82-0.97). We did not observe significant interaction from other interventions, or overall bundle compliance, meaning these interventions' association with mortality did not significantly differ between HPS versus EDPS.

The implied attributable risk difference from discrepancies in initial resuscitation was $23.3 \%$ for mortality, $35.2 \%$ for mechanical ventilation, and $7.6 \%$ for ICU admission (Figure 2B). Resuscitation explained $26.5 \%$ of longer ICU LOS and $16.7 \%$ of longer hospital LOS associated with HPS.

Figure 2C shows sensitivity analysis excluding ICU-presenting sepsis from propensity matching (ie, limiting HPS to hospital ward presentations). Again, HPS was associated with all adverse outcomes, though effect sizes were smaller than in the primary cohort for all outcomes except hospital LOS. In this cohort, resuscitation factors now explained $16.5 \%$ of HPS' association with mortality, and $14.5 \%$ of the association with longer ICU LOS. However, they explained a greater proportion (13.0\%) of ICU admissions. Attributable risk differences were comparable to the primary cohort for mechanical ventilation (37.6\%) and hospital LOS (15.3\%).

\section{DISCUSSION}

In this analysis of 11,182 sepsis and septic shock patients, HPS contributed $22 \%$ of prevalence but $>35 \%$ of total sepsis mortalities, ICU utilization, and hospital days. HPS patients had higher comorbidity burdens and had clinical presentations less obviously attributable to infection with more severe organ dysfunction. EDPS received antibiotics within three hours about 1.62 times more often than do HPS patients. EDPS patients also receive fluids initiated within two hours about 1.82 times more often than HPS patients do. HPS had nearly 1.5-fold greater mortality and LOS, and nearly two-fold greater mechanical ventilation and ICU utilization. Resuscitation disparities could partially explain these associations. These patterns persisted when comparing only wards presenting HPS with EDPS.

Our analysis revealed several notable findings. First, these data confirm that HPS represents a potentially high-impact target population that contributes adverse outcomes disproportionately frequently with respect to case prevalence.

Our findings, unsurprisingly, revealed HPS and EDPS reflect dramatically different patient populations. We found that the two groups significantly differed by the majority of the baseline factors we compared. It may be worth asking if and how these substantial differences in illness etiology, chronic health, and acute physiology impact what we consider an optimal approach to management. Significant interaction effects of fluid volume on the association between HPS and mortality suggest differential treatment effects may exist between patients. Indeed, patients who newly arrive from the community and those who are several days into admission likely have different volume status. However, no interactions were noted with other bundle elements, such as timeliness of antibiotics or timeliness of initial fluids.
Another potentially concerning observation was that HPS patients were admitted much less frequently from skilled nursing facilities, as it could imply that this poorer-fairing population had a comparatively higher baseline functional status. The fact that $25 \%$ of EDPS cases were admitted from these facilities also underscores the need to engage skilled nursing facility providers in future sepsis initiatives.

We found marked disparities in resuscitation. Timely delivery of interventions, such as antibiotics and initial fluid resuscitation, occurred less than half as often for HPS, especially on hospital wards. While evidence supporting the efficacy of specific 3-hour bundle elements remains unsettled, ${ }_{19}^{19}$ a wealth of literature demonstrates a correlation between bundle uptake and decreased sepsis mortality, especially for early antibiotic administration. ${ }^{13,20-26}$ Some analysis suggests that differing initial resuscitation practices explain different mortality rates in the early goal-directed therapy trials. ${ }^{27}$ The comparatively poor performance for non-ICU HPS indicates further $\mathrm{Q}$ l efforts are better focused on inpatient wards, rather than on EDs or ICUs where resuscitation is already delivered with substantially greater fidelity.

While resuscitation differences partially explained outcome discrepancies between groups, they did not account for as much variation as expected. Though resuscitation accounted for $>35 \%$ of attributable mechanical ventilation risk, it explained only $16.5 \%$ of mortality differences for non-ICU HPS vs EDPS. We speculate that several factors may contribute.

First, HPS patients are already hospitalized for another acute insult and may be too physiologically brittle to derive equal benefit from initial resuscitation. Some literature suggests protocolized sepsis resuscitation may paradoxically be more effective in milder/earlier disease. ${ }^{28}$

Second, clinical information indicating septic organ dysfunction may become available too late in HPS - a possible data limitation where inpatient providers are counterintuitively more likely to miss early signs of patients' deterioration and a subsequent therapeutic window. Several studies found that fluid resuscitation is associated with improved sepsis outcomes only when it is administered very early. ${ }^{11,29-31}$ In inpatient wards, decreased monitoring ${ }^{32}$ and human factors (eg, hospital workflow, provider-to-patient ratios, electronic documentation burdens) ${ }^{33,34}$ may hinder early diagnosis. In contrast, ED environments are explicitly designed to identify acutely ill patients and deliver intervention rapidly. If HPS patients were sicker when they were identified, this would also explain their more severe organ dysfunctions. Our data seems to support this possibility. HPS patients had tachypnea less frequently but more often had impaired gas exchange. This finding may suggest that early tachypnea was either less often detected or documented, or that it had progressed further by the time of detection.

Third, inpatients with sepsis may more often present with greater diagnostic complexity. We observed that HPS patients were more often euthermic and less often tachypneic. Beyond suggesting a greater diagnostic challenge, this also raises questions as to whether differences reflect patient physiology (response to infection) or iatrogenic factors (eg, prior antipyret- 
ics). Higher comorbidity and acute physiological burdens also limit the degree to which new organ dysfunction can be clearly attributed to infection. We note differences in the proportion of patients who received antibiotics increased over time, suggesting that HPS patients who received delayed antibiotics did so much later than their EDPS counterparts. This lag could also arise from diagnostic difficulty.

All three possibilities highlight a potential lead time effect, where the same measured three-hour period on the wards, between meeting sepsis criteria and starting treatment, actually reflects a longer period between (as yet unmeasurable) pathobiologic "time zero" and treatment versus the ED. The time of sepsis detection, as distinct from the time of sepsis onset, therefore proves difficult to evaluate and impossible to account for statistically.

Regardless, our findings suggest additional difficulty in both the recognition and resuscitation of inpatient sepsis. Inpatients, especially with infections, may need closer monitoring. How to cost effectively implement this monitoring is a challenge that deserves attention.

A more rational systems approach to HPS likely combines efforts to improve initial resuscitation with other initiatives aimed at both improving monitoring and preventing infection.

To be clear, we do not imply that timely initial resuscitation does not matter on the wards. Rather, resuscitation-focused QI alone does not appear to be sufficient to overcome differences in outcomes for HPS. The $23.3 \%$ attributable mortality risk we observed still implies that resuscitation differences could explain nearly one in four excess HPS mortalities. We previously showed that timely resuscitation is strongly associated with better outcomes. ${ }^{11,13,30}$ As discussed above, the unclear degree to which better resuscitation is a marker for more obvious presentations is a persistent limitation of prior investigations and the present study.

Taken together, the ultimate question that this study raises but cannot answer is whether the timely recognition of sepsis, rather than any specific treatment, is what truly improves outcomes.

In addition to those above, this study has several limitations. Our study did not differentiate HPS with respect to patients admitted for noninfectious reasons and who subsequently became septic versus nonseptic patients admitted for an infection who subsequently became septic from that infection. Nor could we discriminate between missed ED diagnoses and true delayed presentations. We note distinguishing these entities clinically can be equally challenging. Additionally, this was a propensity-matched retrospective analysis of an existing sepsis cohort, and the many limitations of both retrospective study and propensity matching apply. ${ }^{35,36}$ We note that randomizing patients to develop sepsis in the community versus hospital is not feasible and that two of our aims intended to describe overall patterns rather than causal effects. We could not ascertain robust measures of severity of illness (eg, SOFA) because a real world setting precludes required data points-eg, urine output is unreliably recorded. We also note incomplete overlap between inclusion criteria and either Sepsis- 2 or -3 defi- nitions, ${ }^{1,37}$ because we designed and populated our database prior to publication of Sepsis-3. Further, we could not account for surgical source control, the appropriateness of antimicrobial therapy, mechanical ventilation before sepsis onset, or most treatments given after initial resuscitation.

In conclusion, hospital-presenting sepsis accounted for adverse patient outcomes disproportionately to prevalence. HPS patients had more complex presentations, received timely antibiotics half as often ED-presenting sepsis, and had nearly twice the mortality odds. Resuscitation disparities explained roughly $25 \%$ of this difference.

Disclosures: The authors have no conflicts of interest to disclose.

Funding: This investigation was funded in part by a grant from the Center for Medicare and Medicaid Innovation to the High Value Healthcare Collaborative of which the study sites' umbrella health system was a part. This grant helped fund the underlying $\mathrm{Q}$ l program and database in this study.

\section{References}

1. Singer M, Deutschman CS, Seymour CW, et al. The third international consensus definitions for sepsis and septic shock (Sepsis-3). JAMA. 2016;315(8):801810. doi: 10.1001/jama.2016.0287.

2. Torio CMA, Andrews RMA. National inpatient hospital costs: the most expensive conditions by payer, 2011. In. Statistical Brief No. 160. Rockville, MD: Agency for Healthcare Research and Quality; 2013.

3. Liu V, Escobar GJ, Greene JD, et al. Hospital deaths in patients with sepsis from 2 independent cohorts. JAMA. 2014;312(1):90-92. doi: 10.1001/ jama.2014.5804.

4. Seymour CW, Liu VX, Iwashyna TJ, et al. Assessment of clinical criteria for sepsis: for the third international consensus definitions for sepsis and septic shock (Sepsis-3). JAMA. 2016;315(8):762-774. doi: 10.1001/jama.2016.0288.

5. Jones SL, Ashton CM, Kiehne LB, et al. Outcomes and resource use of sepsis-associated stays by presence on admission, severity, and hospital type. Med Care. 2016;54(3):303-310. doi: 10.1097/MLR.0000000000000481.

6. Page DB, Donnelly JP, Wang HE. Community-, healthcare-, and hospital-acquired severe sepsis hospitalizations in the university healthsystem consortium. Crit Care Med. 2015;43(9):1945-1951. doi: 10.1097/ CCM.0000000000001164.

7. Rothman M, Levy M, Dellinger RP, et al. Sepsis as 2 problems: identifying sepsis at admission and predicting onset in the hospital using an electronic medical record-based acuity score. J Crit Care. 2016;38:237-244. doi: 10.1016/j. jcrc.2016.11.037.

8. Chan P, Peake S, Bellomo R, Jones D. Improving the recognition of, and response to in-hospital sepsis. Curr Infect Dis Rep. 2016;18(7):20. doi: 10.1007/ s11908-016-0528-7.

9. Rhodes A, Evans LE, Alhazzani W, et al. Surviving sepsis campaign: international guidelines for management of sepsis and septic shock: 2016. Crit Care Med. 2017;45(3):486-552. doi: 10.1097/CCM.0000000000002255.

10. Levy MM, Evans LE, Rhodes A. The Surviving Sepsis Campaign Bundle: 2018 Update. Crit Care Med. 2018;46(6):997-1000. doi: 10.1097/ CCM.0000000000003119.

11. Leisman DE, Goldman C, Doerfler ME, et al. Patterns and outcomes associated with timeliness of initial crystalloid resuscitation in a prospective sepsis and septic shock cohort. Crit Care Med. 2017;45(10):1596-1606. doi: 10.1097/ CCM.0000000000002574

12. Leisman DE, Doerfler ME, Schneider SM, Masick KD, D'Amore JA, D'Angelo JK. Predictors, prevalence, and outcomes of early crystalloid responsiveness among initially hypotensive patients with sepsis and septic shock. Crit Care Med. 2018;46(2):189-198. doi: 10.1097/CCM.0000000000002834.

13. Leisman DE, Doerfler ME, Ward MF, et al. Survival benefit and cost savings from compliance with a simplified 3-hour sepsis bundle in a series of prospective, multisite, observational cohorts. Crit Care Med. 2017;45(3):395-406. doi: 10.1097/CCM.0000000000002184

14. Doerfler ME, D'Angelo J, Jacobsen D, et al. Methods for reducing sepsis mortality in emergency departments and inpatient units. Jt Comm J Qual Patient Saf. 2015;41(5):205-211. doi: 10.1016/S1553-7250(15)41027-X.

15. Murphy B, Fraeman $\mathrm{KH}$. A general $\mathrm{SAS} \otimes$ macro to implement optimal $\mathrm{N}: 1$ 
propensity score matching within a maximum radius. In: Paper 812-2017. Waltham, MA: Evidera; 2017. https://support.sas.com/resources/papers/ proceedings17/0812-2017.pdf. Accessed February 20, 2019.

16. Funk MJ, Westreich D, Wiesen C, Stürmer T, Brookhart MA, Davidian M. Dou bly robust estimation of causal effects. Am J Epidemiol. 2011;173(7):761-767. doi: 10.1093/aje/kwq439.

17. Sahai HK, Khushid A. Statistics in Epidemiology: Methods, Techniques, and Applications. Boca Raton, FL: CRC Press; 1995.

18. VanderWeele TJ. On a square-root transformation of the odds ratio for a common outcome. Epidemiology. 2017;28(6):e58-e60. doi: 10.1097/ EDE.0000000000000733

19. Pepper DJ, Natanson C, Eichacker PQ. Evidence underpinning the centers for medicare \& medicaid services' severe sepsis and septic shock management bundle (SEP-1). Ann Intern Med. 2018;168(8):610-612. doi: 10.7326/ L18-0140.

20. Levy MM, Rhodes A, Phillips GS, et al. Surviving sepsis campaign: association between performance metrics and outcomes in a 7.5-year study. Crit Care Med. 2015;43(1):3-12. doi: 10.1097/CCM.0000000000000723.

21. Liu VX, Morehouse JW, Marelich GP, et al. Multicenter Implementation of a Treatment Bundle for Patients with Sepsis and Intermediate Lactate Values. Am J Respir Crit Care Med. 2016;193(11):1264-1270. doi: 10.1164/rccm.201507-14890C.

22. Miller RR, Dong L, Nelson NC, et al. Multicenter implementation of a severe sepsis and septic shock treatment bundle. Am J Respir Crit Care Med. 2013;188(1):77-82. doi: 10.1164/rccm.201212-21990C

23. Seymour CW, Gesten F, Prescott HC, et al. Time to treatment and mortality during mandated emergency care for sepsis. $N$ Engl J Med. 2017;376(23):2235-2244. doi: 10.1056/NEJMoa1703058.

24. Pruinelli L, Westra BL, Yadav $P$, et al. Delay within the 3-hour surviving sepsis campaign guideline on mortality for patients with severe sepsis and septic shock. Crit Care Med. 2018:46(4):500-505. doi: 10.1097/ CCM.0000000000002949.

25. Kumar A, Roberts D, Wood KE, et al. Duration of hypotension before initiation of effective antimicrobial therapy is the critical determinant of survival in human septic shock. Crit Care Med. 2006;34(6):1589-1596. doi: 10.1097/01. CCM.0000217961.75225.E9.

26. Liu VX, Fielding-Singh V, Greene JD, et al. The timing of early antibiotics and hospital mortality in sepsis. Am J Respir Crit Care Med. 2017;196(7):856-863. doi: 10.1164/rccm.201609-1848OC.

27. Kalil AC, Johnson DW, Lisco SJ, Sun J. Early goal-directed therapy for sepsis: a novel solution for discordant survival outcomes in clinical trials. Crit Care Med. 2017;45(4):607-614. doi: 10.1097/CCM.0000000000002235.

28. Kellum JA, Pike F, Yealy DM, et al. relationship between alternative resuscitation strategies, host response and injury biomarkers, and outcome in septic shock: analysis of the protocol-based care for early septic shock study. Crit Care Med. 2017;45(3):438-445. doi: 10.1097/CCM.0000000000002206.

29. Seymour CW, Cooke CR, Heckbert SR, et al. Prehospital intravenous access and fluid resuscitation in severe sepsis: an observational cohort study. Crit Care. 2014;18(5):533. doi: 10.1186/s13054-014-0533-x.

30. Leisman $D$, Wie $B$, Doerfler $M$, et al. Association of fluid resuscitation initiation within 30 minutes of severe sepsis and septic shock recognition with reduced mortality and length of stay. Ann Emerg Med. 2016;68(3):298-311. doi: 10.1016/j.annemergmed.2016.02.044.

31. Lee SJ, Ramar K, Park JG, Gajic O, Li G, Kashyap R. Increased fluid administration in the first three hours of sepsis resuscitation is associated with reduced mortality: a retrospective cohort study. Chest. 2014;146(4):908-915. doi: 10.1378/chest.13-2702

32. Smyth MA, Daniels R, Perkins GD. Identification of sepsis among ward patients. Am J Respir Crit Care Med. 2015;192(8):910-911. doi: 10.1164/rc$\mathrm{cm} \cdot 201507-1395 \mathrm{ED}$

33. Wenger N, Méan M, Castioni J, Marques-Vidal P, Waeber G, Garnier A. Allocation of internal medicine resident time in a Swiss hospital: a time and motion study of day and evening shifts. Ann Intern Med. 2017;166(8):579-586. doi: 10.7326/M16-2238.

34. Mamykina L, Vawdrey DK, Hripcsak G. How do residents spend their shift time? A time and motion study with a particular focus on the use of computers. Acad Med. 2016;91(6):827-832. doi: 10.1097/ACM.0000000000001148.

35. Kaji AH, Schriger D, Green S. Looking through the retrospectoscope: reducing bias in emergency medicine chart review studies. Ann Emerg Med. 2014;64(3):292-298. doi: 10.1016/j.annemergmed.2014.03.025.

36. Leisman DE. Ten pearls and pitfalls of propensity scores in critical care research: a guide for clinicians and researchers. Crit Care Med. 2019;47(2):176185. doi: 10.1097/CCM.0000000000003567.

37. Levy MM, Fink MP, Marshall JC, et al. 2001 SCCM/ESICM/ACCP/ATS/SIS international sepsis definitions conference. Crit Care Med. 2003;31(4):12501256. doi: 10.1097/01 CCM.0000050454.01978.3B 